\title{
Warped Accretion Discs
}

\author{
D. Chakrabarty, ${ }^{a}$ J. Murray, G. A. Wynn and A. R. King \\ Theoretical Astrophysics Group, Department of Physics and Astronomy, \\ University of Leicester, Leicester, U.K. \\ a dac@kaam.star.le.ac.uk
}

\begin{abstract}
In this article we report the results of our numerical investigation of warped accretion discs in binary stellar systems. We perform complete 3-D hydrodynamic simulations of binary discs. The disc is rendered unstable to the warp mode under the action of the magnetic field of the companion star in the binary. The disc thus warped is noted to undergo retrograde precession with a precession period just slightly less than the binary period. This small difference in periods can explain the phenomenon of negative superhumps observed in a number of binaries. Besides the modal analysis based on Fourier transforms, warps were also studied by a simple and robust technique that we developed; this is based on an analysis of the azimuthal distributions of particles that lie above and below the mid-plane of the disc.
\end{abstract}

\section{Magnetically driven warp}

Observations of extra periodicities in systems with accretion discs have indicated in certain cases that the discs are warped. Accretion discs in binary stellar systems are expected to warp under the effects of either one or more of the following; radiation from the central source (Pringle, 1996), disc winds and magnetic fields. We have successfully demonstrated that accretion discs warp into the equatorial plane, under the influence of the magnetic field of the companion star in a binary system. The disc thus warped, is noted to undergo retrograde precession with periods slightly lower than the binary orbital period $\tau_{b}$. We carried out a completely three dimensional hydrodynamical modelling of discs in the full potential of the binary (Murray et. al, 2001). The number of particles in this simulation is 536033 and the used mass ratio is 0.4 . The simulations were carried out using UKAFF (United Kingdom Astrophysical Fluids Facility) which is sited at the University of Leicester.

The light curves from a number of systems have been reported to exhibit negative superhumps, i.e. amplitude modulation on periods slightly lower than $\tau_{b}$ (Patterson, 1999). Our simulations establish one possible origin of this phenomenon.

\section{Characterisation of warps}

Along with the modal analysis based on Fourier transforms, we used a robust and simple technique that we have developed to identify warps in discs and 
estimate the periodicity of the warp mode. This method is based on the two different azimuthal distributions of particles that lie above and below the midplane at given radii and time (Chakrabarty et. al, 2001). These distributions are referred to as the $z_{+v e}$ and the $z_{-v e}$ distributions respectively. We had to extract one statistic from such histograms which would realistically represent the characteristics of the warp. The centroid of an azimuthal distribution was chosen as this quantity. The temporal variations of the centroids of the two distributions are plotted; for a neatly warped disc the phase difference between the centroid evolution plots corresponding to the $z_{+v e}$ and $z_{-v e}$ histograms is expected to be $\pi$.

Chakrabarty et. al, 2001 report a study of the long-term evolution of warps in binary accretion discs using the technique described above. A series of simulations are performed with discs warped "by hand" in order to check the strength of the developed method. In these simulations the disc is either tilted through an angle (which subsequently leads to it being warped under the effects of viscosity) or the particles of the incoming stream are instructed to take up appropriate $z$ values to ensure a pre-fixed uniform tilt angle. A neat warp is clearly borne by these simulations. The paper quotes precessional period of the disc as a function of the initial system configuration as well as the decay time of the warp subject to the diluting effects of viscosity. In this work, magnetically induced warps are also investigated. Such modes are identified as complex structures which require a significant time to develop.

\section{Conclusion}

We have successfully demonstrated that warps can be generated by the magnetic field of the secondary and can survive over long times. The magnetically induced warps were noted to have periods which were slightly less than the binary orbital period, indicating retrograde precession of the disc. This is a ready explanation for negative superhumps. As expected, the amplitude of the warp decays on the viscous time-scale. The technique that we introduce in this article is a viable way of identifying characteristics of a warp; it appears to pick up even the weakest warps in discs. We suggest wider applications of this technique in other disc systems which have undergone the warp instability.

\section{References}

Patterson, J. 1999, in Disk Instabilities in Close Binary Systems, ed.s S. Mineshige \& J.C. Wheeler, Universal Academy Press, 61

Pringle, J.E. 1996, MNRAS, 281, 357

Murray, J. R., Chakrabarty, Dalia, Wynn, G. A., \& Kramer L. 2001, submitted to MNRAS

Chakrabarty, Dalia, Murray, J. R., \& Wynn, G. A. 2001, submitted to MNRAS 